\title{
Correction to: The short form of Mendeleev's Periodic Table of Chemical Elements: toolbox for learning the basics of inorganic chemistry. A contribution to celebrate 150 years of the Periodic Table in 2019
}

\author{
Alexander A. Andriiko ${ }^{1}$. Hans-Joachim Lunk ${ }^{2}$ \\ Published online: 23 April 2020 \\ (c) Springer Nature Switzerland AG 2020
}

\section{Correction to: ChemTexts (2018) 4:4 \\ https://doi.org/10.1007/s40828-018-0059-y}

We regret that Table 2 did not list all 118 elements of Mendeleev's Periodic Table.

The modified Table 2 presents seven periods of discovery, where the elements of each period are placed according to their discovery year.

The original article can be found online at https://doi.org/10.1007/ s40828-018-0059-y.

\section{Alexander A. Andriiko} andriiko_aa@ukr.net

1 Igor Sikorskiy National Technical University of Ukraine 'Kyiv Polytechnic Institute', 37 Peremogy av., Kiev 03057, Ukraine

22858 Lake RD, Towanda, PA 18848, USA 
Table 2 Suggested seven periods of discovery of the 118 elements of the Periodic Table

Elements known to humans since ancient times*

$\left(8000 \mathrm{BCE}^{\#}\right)(\mathrm{Cu})-800 \mathrm{BCE}(\mathrm{Sb}) ; 7200$ years: 8 elements

Copper (Cu), Lead (Pb), Gold (Au), Silver (Ag), Tin (Sn), Sulfur (S), Mercury (Hg), Antimony (Sb)

$\sim 1400$ (Bi)-1797 (V); 400 years: 24 elements

Bismuth (Bi), Phosphorus (P), Carbon (C), Iron (Fe), Platinum (Pt), Nickel (Ni), Cobalt (Co), Magnesium (Mg), Nitrogen (N), Oxygen (O), Chlorine (Cl), Chromium (Cr), Manganese (Mn), Hydrogen (H), Molybdenum (Mo), Tellurium(Te), Tungsten (W), Zirconium(Zr), Uranium (U), Strontium (Sr), Scandium (Sc), Yttrium (Y), Beryllium (Be), Vanadium (V)

$1801(\mathrm{Nb})-1844(\mathrm{Ru}) ; 44$ years: 24 elements

Niobium (Nb), Tantalum (Ta), Palladium (Pd), Cerium (Ce), Osmium (Os), Iridium (Ir), Rhodium (Rh), Potassium (K), Sodium (Na), Barium (Ba), Boron (B), Iodine (I), Lithium (Li), Cadmium (Cd), Silicon (Si), Arsenic (As), Aluminum (Al), Selenium (Se), Thorium (Th), Titanium (Ti), Lanthanum (La), Erbium (Er), Terbium (Tb), Ruthenium (Ru)

1860 (Cs)-1900 (Rn); 41 years: 26 elements

Caesium (Cs), Thallium (Tl), Rubidium (Rb), Indium (In), Holmium (Ho), Zinc (Zn), Ytterbium (Yb), Calcium (Ca), Samarium (Sm), Thulium (Tm), Gadolinium (Gd), Praseodymium (Pr), Neodymium (Nd), Germanium (Ge), Gallium (Ga), Dysprosium (Dy), Fluorine (F), Argon (Ar), Helium (He), Krypton (Kr), Neon (Ne), Xenon (Xe), Polonium (Po), Radium (Ra), Actinium (Ac), Radon (Rn)

$1901(\mathrm{Eu})-1945(\mathrm{Pr}) ; 45$ years: 13 elements

Europium (Eu), Lutetium (Lu), Protactinium (Pa), Hafnium (Hf), Rhenium (Re), Technetium (Tc), Francium (Fr), Astatine (At), Neptunium $(\mathrm{Np})$, Plutonium $(\mathrm{Pu})$, Curium $(\mathrm{Cm})$, Americium $(\mathrm{Am})$, Promethium $(\mathrm{Pm})$

1949 (Bk)-1984 (Hs); 36 years: 13 elements

Berkelium (Bk), Californium (Cf), Einsteinium (Es), Fermium (Fm), Mendelevium (Md), Nobelium (No), Lawrencium (Lr), Rutherfordium (Rf), Dubnium (Db), Seaborgium (Sg), Bohrium (Bh), Meitnerium (Mt), Hassium (Hs)

1994 (Ds/Rg)-2010 (Ts); 17 years; 9 elements

Darmstadtium (Ds), Roentgenium (Rg), Copernicium (Cn), Nihonium (Nh), Oganesson (Og), Flerovium (Fl), Livermorium (Lv), Moscovium $(\mathrm{Mc})$, Tennessine (Ts)

(cf. Sec. 'Early classifications of the chemical elements')

*At that time not understood as elements in the modern definition

${ }^{\#}$ BCE (Before Common Era) and BC (Before Christ) mean the same: previous to year 1 CE (Common Era)

Publisher's Note Springer Nature remains neutral with regard to jurisdictional claims in published maps and institutional affiliations. 